\title{
INTEGRIDADE DO MÚSCULO SUBESCAPULAR APÓS A CIRURGIA ABERTA PARA TRATAMENTO DA LUXAÇÃO RECIDIVANTE GLENOUMERAL: AVALIAÇÃO CLÍNICA E RADIOLÓGICA
}

\author{
INTEGRITY OF THE SUBSCAPULARIS TENDON AFTER OPEN SURGERY FOR THE TREATMENT \\ OF ANTERIOR SHOULDER INSTABILITY: A CLINICAL AND RADIOLOGICAL EVALUATION
}

Osvandré Lech', Paulo Piluski ${ }^{2}$, Renato Tambani ${ }^{3}$, Nero Castro ${ }^{4}$, Gilnei Pimentel ${ }^{5}$

\section{RESUMO}

Objetivo: Avaliar a integridade do músculo subescapular através da força, função e ressonância nuclear magnética após acesso deltopeitoral para tratamento da luxação glenoumeral anterior recidivante. Métodos: Foram avaliados 20 pacientes com luxação recidivante do ombro. Todos os casos possuíam seguimento mínimo de 12 meses, com média de 40 meses. Os pacientes eram todos do sexo masculino, com média de idade de 29 anos (20-42 anos). Os pacientes foram submetidos a exame físico para avaliar mobilidade, força muscular, "Belly" Teste e teste de Gerber. A força isocinética em rotação interna e externa, em velocidades angulares de $60 \%$ s e $180 \%$, em ambos os ombros, foi medida utilizando-se um dinamômetro. Em 15 pacientes foi realizada ressonância nuclear magnética (RNM) em ambos os ombros para avaliação da espessura, área e possível hipotrofia do músculo subescapular. Resultados: Houve diferença significativa entre os picos de torque para a velocidade de $60 \%$ para as rotações interna $(p=0,036)$ e externa $(p=0,008)$. Porém, para a velocidade de $180^{\circ}$ s a situação se inverte (rotação interna: $\mathrm{p}$ $=0,133$; rotação externa: $\mathrm{p}=0,393$ ). A espessura e a área do subescapular são significativamente menores que as do lado normal, com déficit de $19 \%$ e $23 \%$, respectivamente. De acordo com os escores de Rowe e da UCLA, observamos excelentes e bons resultados na maioria dos pacientes, com média de 88 e 31,6 pontos, respectivamente. Conclusão: Apesar dos bons resultados funcionais, a cirurgia aberta pode restringir a força, bem como reduzir a espessura e a área da seção transversal do músculo subescapular. Os melhores resultados ocorreram no lado dominante.

Descritores - Instabilidade glenoumeral; Subescapular; Ressonância nuclear magnética

\section{ABSTRACT}

Objective: To evaluate the integrity of the subscapularis tendon by strength, function and magnetic resonance imaging after deltopectoralis access for anterior shoulder instability. Methods: 20 patients with anterior shoulder instability have been evaluated. Minimum follow-up was 12 months, with a mean of 40 months. Only male patients were included, with a mean of age of 29 years (20 - 42 years). The patients have been submitted to physical examinations of mobility, muscular strength, Belly Test and Gerber Test. The isokinetic strength in internal and external rotation, in angular speeds of $60 \%$ and $180 \%$, for both shoulders was measured using a dynamometer. In 15 patients magnetic resonance imaging (MRI) was carried out on both shoulders for evaluating the thickness, cross-sectional area and atrophy of the subscapularis muscle. Results: A significant difference was found between torque peaks at the speed of $60 \%$ for internal $(p=0.036)$ and external $(p=0.008)$ rotation. However, at $180 \%$ s the opposite happens (internal rotation: $p=0.133$; external rotation: $p=0.393$ ). Subscapularis muscle thickness and area are significantly smaller than the normal side, with a deficit of $19 \%$ and $23 \%$, respectively. According to Rowe and UCLA scores, we find excellent and good results for the majority of patients, with a mean of 88 and 31.6 points, respectively. Conclusion: Despite of the good functional results, open surgery can limit strength and reduce the thickness and the cross-sectional area of subscapularis muscle. However, the best results were found in the patients who had the dominant side operated.

Keywords - Anterior shoulder instability; Subscapularis; Magnetic resonance imaging

\footnotetext{
1 - Chefe do Serviço de Residência Médica e do Treinamento Pós-Residência do Instituto de Ortopedia e Traumatologia, Passo Fundo, RS, Brasil.

2 - Instrutor da Residência Médica do Instituto de Ortopedia e Traumatologia, Passo Fundo, RS, Brasil.

3 - Médico Residente (R4) do Serviço de Cirurgia do Ombro e Cotovelo do Instituto de Ortopedia e Traumatologia de Passo Fundo, RS, Brasil.

4 - Médico Radiologista da Clínica Kozma, Passo Fundo, RS, Brasil.

5 - Professor do Curso de Fisioterapia da Universidade de Passo Fundo, RS, Brasil.
}

Correspondência: Instituto de Ortopedia Traumatologia, Rua Uruguai, 2.050 - 90.010-112 - Passo Fundo, RS. E-mail: ensino@iotrs.com.br 


\section{INTRODUÇÃO}

As luxações na articulação glenoumeral são mais frequentes do que as que ocorrem em qualquer outra articulação e a deformidade e o comprometimento da função que surgem com uma luxação de ombro são amplamente conhecidos. As primeiras evidências de ocorrência de luxação são encontradas nas pinturas das tumbas do antigo Egito. Indubitavelmente, os homens das cavernas pré-históricos também reconheciam essas lesões ${ }^{(1)}$.

As luxações podem ser classificadas em traumáticas, atraumáticas e recidivantes. A traumática é causada por força violenta sobre a articulação glenoumeral, que leva geralmente a uma lesão do lábio, ligamentos glenoumerais e cápsula - denominado de lesão de Bankart ${ }^{(2,3)}$. A ocorrência das luxações traumáticas da articulação glenoumeral é relativamente comum, principalmente a anterior, que acomete 1,5 a $2 \%$ da população em geral e em torno de $7 \%$ de atletas que utilizam o membro superior. A incidência é mais frequente em indivíduos jovens, que realizam alguma prática esportiva e menos comum em mais velhos, onde as lesões mais comuns são as fraturas ou lesões do manguito rotador ${ }^{(3)}$. O tipo atraumático ocorre por episódios de subluxação sem trauma. A articulação possui uma cápsula hipermóvel, permitindo movimento excessivo de translação. A subluxação pode ser anterior, posterior ou inferior, sendo que na maioria dos casos existe um somatório de direções, constituindose uma instabilidade bidirecional ou multidirecional ${ }^{(2-4)}$. O tipo recidivante ocorre após traumatismos menores. Em torno de $80 \%$ dos casos de luxação traumática primária evoluem para recidivas. Quanto mais intensos são os episódios de luxação, mais frequente será a recidiva, devido ao aumento progressivo da lesão, que acontece com as sucessivas luxações ${ }^{(2-4)}$.

A lesão de Bankart é a desinserção da porção anterior da cápsula articular e do lábio junto ao rebordo da glenoide. Essa lesão ocorre na luxação escapuloumeral anterior e está presente em aproximadamente 85\% das luxações recidivantes. O entendimento da avulsão do lábio, conhecido como lesão de Bankart ou lesão “essencial”, foi fundamental para o aprimoramento da técnica cirúrgica ${ }^{(5,6)}$.

O conhecimento da anatomia e biomecânica do ombro melhorou consideravelmente nos últimos anos com a ressonância magnética (RM), a artrorressonância (artro-RM), os estudos laboratoriais biomecânicos mais detalhados e o com o uso da artroscopia. Os avanços dos métodos diagnósticos e o conhecimento anatômico per- mitiram que novas lesões associadas fossem reveladas, tais como: SLAP (superior labrum anterior and posterior), GLAD (glenoid labrum articular disruption), ALPSA (anterior labroligamentous periosteal sleeve avulsion), HAGL (humeral avulsion of the glenohumeral ligament), modificando o método de tratamento ${ }^{(7)}$.

O emprego das âncoras de sutura diminuiu o tempo cirúrgico e facilitou tecnicamente o procedimento. O entendimento dos conceitos de instabilidade e de frouxidão capsuloligamentar foi igualmente importante na escolha da técnica apropriada. As técnicas cirúrgicas atuais visam à reconstrução das lesões associadas, sem causar restrição da mobilidade ou de atividade do paciente ${ }^{(8)}$.

O tratamento da luxação recidivante do ombro pode ser feito pelo método convencional, ou seja, a cirurgia aberta, ou pelo método artroscópico. Os resultados do tratamento da luxação recidivante por via convencional, segundo a literatura, apresentam índices de recidivas que variam entre 3 e $8 \%$ dependendo do autor, sendo considerada ainda golden standard para essa lesão ${ }^{(2,5)}$. A artroscopia trouxe grandes avanços, como melhor conhecimento das lesões associadas, melhor estética, menor risco de falência do músculo subescapular, rápido retorno ao trabalho e esportes e alta satisfação do paciente ${ }^{(6,8,9,10)}$. Apresenta resultados semelhantes aos da cirurgia aberta quando uma adequada seleção do paciente é realizada ${ }^{(8,9)}$.

O objetivo deste trabalho é avaliar clínica e radiologicamente a integridade pós-operatória do músculo subescapular através da força, função e ressonância nuclear magnética após acesso deltopeitoral para o tratamento da luxação glenoumeral anterior recidivante.

\section{MÉTODOS}

De março de 1997 a novembro de 2006, 96 pacientes com luxação recidivante anterior do ombro foram operados por acesso deltopeitoral com inserção de âncoras no Serviço de Ombro e Cotovelo do Instituto de Ortopedia e Traumatologia de Passo Fundo-RS (IOT); desses, 20 casos retornaram para a avaliação do estudo. Todos os casos possuíam seguimento mínimo de 12 meses e máximo de 128, com média de 40 meses. Os pacientes eram todos do sexo masculino, com média de idade de 29 anos (20-42 anos), altura média de 1,76m (1,67-1,89m) e peso médio de $76,1 \mathrm{~kg}$ (59-103kg). O lado dominante foi acometido em 13 pacientes (65\%) (Tabela 1).

Os pacientes foram submetidos a exames físicos de mobilidade, força muscular, "Belly" Teste e teste de Gerber. 
Tabela 1 - Dados dos pacientes

\begin{tabular}{|c|c|c|c|c|c|c|c|c|c|c|}
\hline Caso & Nome & Meses P.O & Sexo & Idade (anos) & Peso (kg) & Altura (m) & Lado operado & Lado dominante & UCLA & Rowe \\
\hline 1 & L.L.F & 20 & M & 37 & 78,0 & 1,71 & direito & direito & 33 & 100 \\
\hline 2 & W.R.L & 20 & $M$ & 22 & 72,0 & 1,72 & esquerdo & esquerdo & 31 & 75 \\
\hline 3 & A.F & 81 & M & 42 & 80,0 & 1,70 & esquerdo & direito & 26 & 75 \\
\hline 4 & J.A.C & 53 & $M$ & 36 & 68,0 & 1,83 & esquerdo & direito & 33 & 90 \\
\hline 5 & P.R.O & 23 & $M$ & 25 & 60,0 & 1,70 & direito & direito & 31 & 100 \\
\hline 6 & C.P & 39 & M & 22 & 76,0 & 1,71 & esquerdo & direito & 35 & 100 \\
\hline 7 & J.S.D & 30 & $M$ & 28 & 59,0 & 1,67 & direito & direito & 31 & 90 \\
\hline 8 & M.G & 128 & $M$ & 32 & 80,0 & 1,83 & direito & direito & 31 & 90 \\
\hline 9 & A.G & 64 & $M$ & 25 & 82,5 & 1,82 & direito & direito & 24 & 70 \\
\hline 10 & A.C.G & 12 & $M$ & 20 & 103,0 & 1,89 & esquerdo & direito & 33 & 100 \\
\hline 11 & M.A.S & 22 & $\mathrm{M}$ & 23 & 68,0 & 1,72 & direito & direito & 31 & 100 \\
\hline 12 & T.P.R & 59 & $\mathrm{M}$ & 23 & 89,0 & 1,75 & esquerdo & direito & 33 & 100 \\
\hline 13 & P.R & 38 & $M$ & 26 & 62,0 & 1,70 & esquerdo & direito & 31 & 90 \\
\hline 14 & L.A & 48 & $\mathrm{M}$ & 36 & 83,0 & 1,83 & direito & direito & 33 & 90 \\
\hline 15 & E.E.C & 40 & $\mathrm{M}$ & 36 & 68,0 & 1,75 & esquerdo & esquerdo & 31 & 90 \\
\hline 16 & D.B.D & 40 & $\mathrm{M}$ & 33 & 84,0 & 1,76 & direito & direito & 31 & 70 \\
\hline 17 & M.A.F & 14 & $\mathrm{M}$ & 26 & 83,0 & 1,79 & direito & direito & 33 & 50 \\
\hline 18 & F.G.L & 29 & $\mathrm{M}$ & 29 & 76,0 & 1,74 & direito & direito & 35 & 100 \\
\hline 19 & A.L.M & 14 & $\mathrm{M}$ & 27 & 72,0 & 1,75 & direito & direito & 33 & 100 \\
\hline 20 & S.G & 32 & M & 25 & 77,5 & 1,81 & esquerdo & direito & 33 & 75 \\
\hline
\end{tabular}

Legendas: M: masculino, F: feminino

Fonte: SAME IOT, Passo Fundo, RS

A força isocinética em rotação interna e externa em ambos os ombros foi mensurada utilizando-se o dinamômetro Biodex Multi Joint System 3 Pro (Laboratório de Biomecânica da Universidade de Passo Fundo, RS) (Figura 1). Para a realização do teste, o paciente foi colocado na posição neutra modificada, na qual fica sentado com o assento inclinado em $15^{0}$, o dinamômetro orientado em $20^{\circ}$ e inclinado $50^{\circ}$. Durante a realização do teste o paciente recebeu estímulo verbal. A força foi testada em velocidades angulares de $60 \%$ s e $180^{\%} / \mathrm{s}^{(11)}$. A primeira sessão foi constituída por três repetições de máximos esforços com velocidade de $60 \%$ s. Após descanso suficiente de 30 segundos, outra sessão de três repetições de máximos esforços com velocidade de $180^{\circ}$ s foi realizada.

Para análises dos dados foram comparadas as diferenças entre as forças isocinéticas medidas nos membros operado e não operado, tanto para a rotação interna e externa e velocidades angulares de $60 \%$ s e $180 \%$ s. Também foram comparados os déficits de força obtidos para a rotação interna e externa para a mesma velocidade. Para melhor compreensão dos resultados, as comparações também foram feitas dividindo-se os pacientes em dois grupos distintos: os com o lado operado dominante e os com o lado operado não dominante.
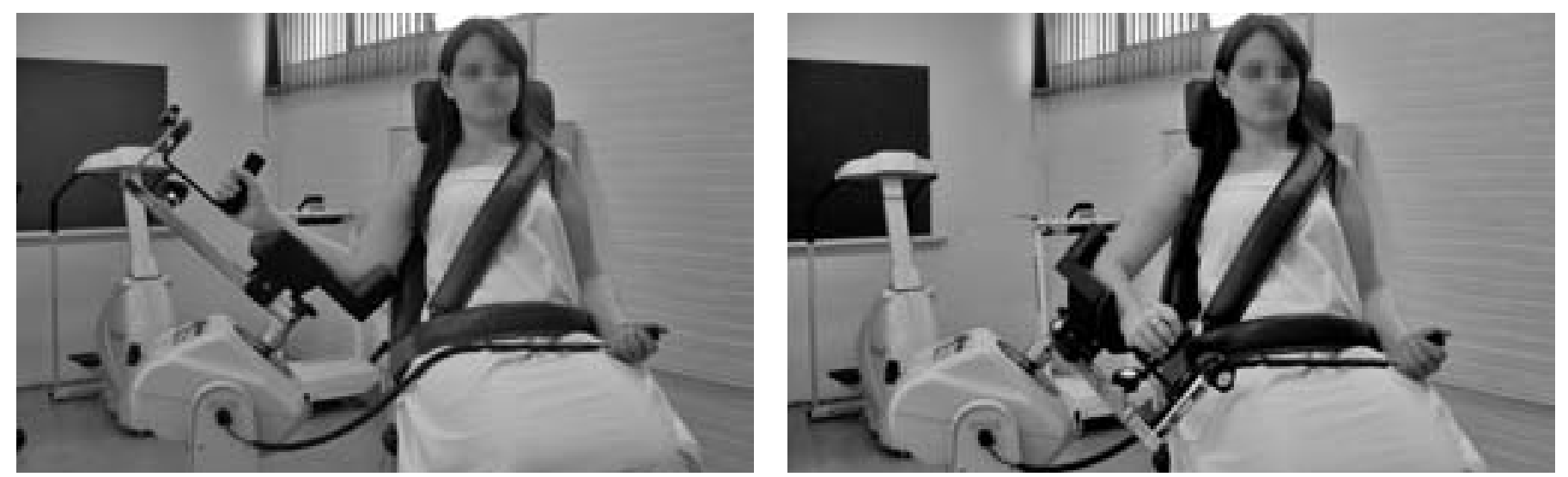

Figura 1 - Posicionamento do paciente no dinamômetro em rotação externa e interna 
Em 15 pacientes foi realizada ressonância nuclear magnética (RNM) em ambos os ombros para avaliação da espessura, da área da seção transversal e da hipotrofia do músculo subescapular ${ }^{(12-14)}$. As imagens foram feitas pelo equipamento Siemens Avanto 1.5 Tesla na clínica radiológica Kozma, em Passo Fundo, RS. Todas as imagens foram obtidas nos planos sagital oblíquo e axial, ponderado em $\mathrm{T}_{1}$ e DPW FAT SAT. A imagem axial escolhida foi a que apresentava claramente o lábio anterior e posterior da glenoide (Figura 2). Nessa imagem foi traçada uma linha paralela ao lábio; nesse mesmo nível, foram mensuradas, em uma imagem sagital (Figura 3), a espessura, a área da seção transversal e a degeneração gordurosa do tendão subescapular. Para a determinação da espessura, uma linha perpendicular ao eixo na parte central do tendão foi traçada conectando as margens anterior e poste-

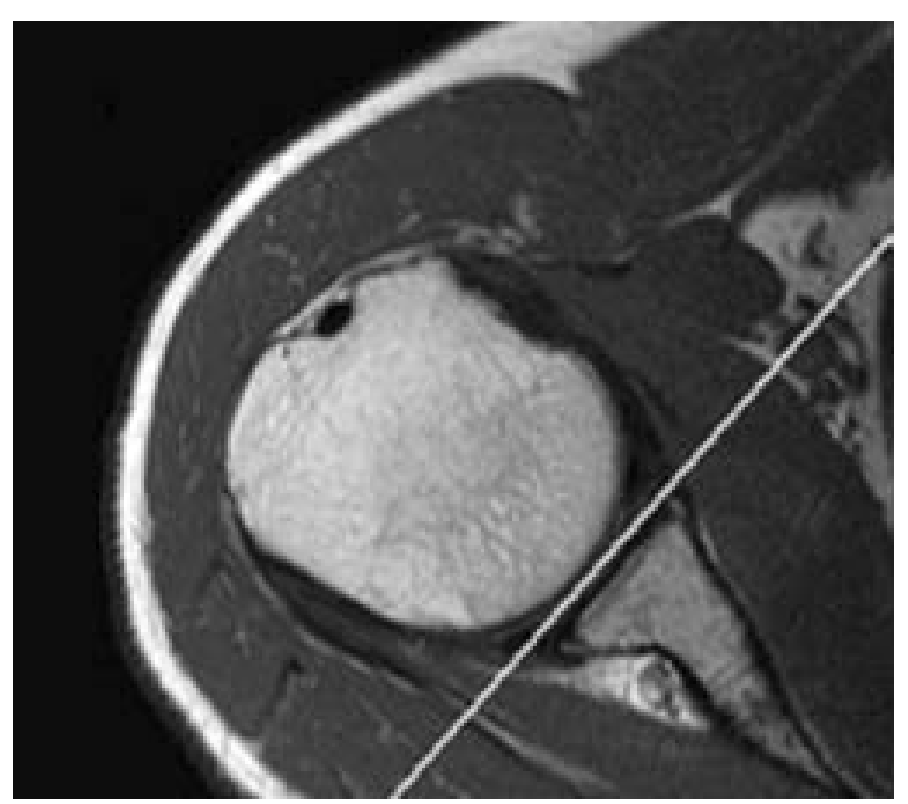

Figura 2 - Imagem da RNM em plano axial rior do mesmo na imagem sagital. A degeneração gordurosa foi avaliada segundo a classificação de Bernageau e Goutallier (Quadro 1). Tanto a espessura como a área da seção transversal foram mensuradas com o auxílio do software analisador de imagens e FilmeLite.

Os sistemas de pontuação de Rowe e da UCLA (University of California at Los Angeles) foram aplicados para avaliação dos pacientes.

A análise estatística dos resultados foi feita através do teste $t$ de Student pareado. Foi considerado estatisticamente significativo quando $\mathrm{p}<0,05$.

Quadro 1 - Classificação de degeneração gordurosa segundo Bernageau e Goutallier

\begin{tabular}{|l|l|}
\hline Grau 0 & Sem presença de gordura \\
\hline Grau 1 & Pequenas estrias de gordura \\
\hline Grau 2 & Proporção menor de gordura em relação ao músculo \\
\hline Grau 3 & Proporção de músculo e gordura se equivale \\
\hline Grau 4 & Proporção de gordura maior que de músculo \\
\hline
\end{tabular}

\section{RESULTADOS}

Todos os pacientes tiveram "Belly" Teste e teste de Gerber negativos.

Os resultados das avaliações de força isocinética estão representados na Tabela 2. Comparando-se os picos de torque obtidos para os ombros operado e não operado, para a velocidade angular de $60 \%$, tanto para a rotação interna $(p=0,036)$ como para a externa $(p=0,008)$, as diferenças encontradas foram estatisticamente significativas. Porém, para a velocidade de $180 \%$ s as reduções nos picos de torque não foram significativas (rotação interna: $\mathrm{p}=0,133$; rotação externa: $\mathrm{p}=0,393$ ).
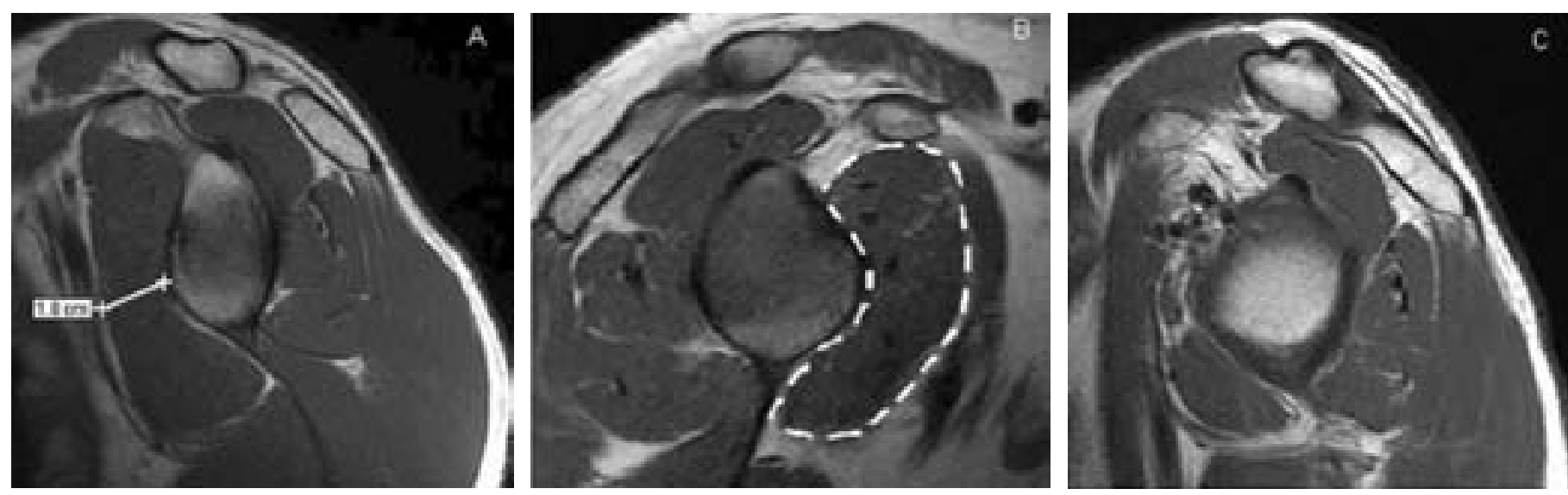

Figura 3 - Imagens das RNM em plano sagital. A) para mensuração da espessura; B) para mensuração da área; e C) para avaliação da degeneração gordurosa 
Tabela 2 - Avaliação da força isocinética

\begin{tabular}{|c|c|c|c|c|c|c|c|c|c|c|c|c|}
\hline \multirow{4}{*}{ Paciente } & \multicolumn{6}{|c|}{$600 / \mathrm{s}$} & \multicolumn{6}{|c|}{$1800 / \mathrm{s}$} \\
\hline & \multicolumn{3}{|c|}{ Rotação externa } & \multicolumn{3}{|c|}{ Rotação interna } & \multicolumn{3}{|c|}{ Rotação externa } & \multicolumn{3}{|c|}{ Rotação interna } \\
\hline & \multicolumn{2}{|c|}{ Pico de torque (N.m) } & \multirow[t]{2}{*}{ Déficit (\%) } & \multicolumn{2}{|c|}{ Pico de torque (N.m) } & \multirow[t]{2}{*}{ Déficit (\%) } & \multicolumn{2}{|c|}{ Pico de torque (N.m) } & \multirow[t]{2}{*}{ Déficit(\%) } & \multicolumn{2}{|c|}{ Pico de torque (N.m) } & \multirow[t]{2}{*}{ Déficit(\%) } \\
\hline & NO & 0 & & NO & 0 & & NO & 0 & & NO & 0 & \\
\hline L.L.F & 54,20 & 55,50 & 2,30 & 58,30 & 68,20 & 17,00 & 50,30 & 53,70 & 6,70 & 62,50 & 71,20 & 13,90 \\
\hline W.R.L & 26,80 & 18,80 & 29,80 & 40,60 & 23,40 & 42,40 & 13,70 & 18,70 & 36,40 & 16,80 & 18,20 & 8,20 \\
\hline A.F & 36,10 & 32,60 & 9,60 & 50,10 & 41,00 & 18,20 & 27,90 & 21,90 & 21,50 & 29,20 & 21,40 & 26,70 \\
\hline J.A.C & 23,40 & 20,20 & 13,60 & 41,90 & 43,00 & 2,70 & 17,70 & 18,90 & 6,70 & 41,30 & 34,00 & 17,60 \\
\hline P.R.O & 25,50 & 19,70 & 22,50 & 36,80 & 24,70 & 32,80 & 21,00 & 17,30 & 17,70 & 22,40 & 24,30 & 8,50 \\
\hline C.P & 36,30 & 31,50 & 13,20 & 50,00 & 37,10 & 25,80 & 18,00 & 23,00 & 27,50 & 24,00 & 28,40 & 18,20 \\
\hline J.S.D & 30,50 & 26,50 & 13,00 & 41,60 & 33,90 & 18,50 & 22,90 & 21,90 & 4,50 & 24,40 & 23,70 & 2,80 \\
\hline M.G & 23,00 & 39,10 & 70,30 & 38,80 & 51,70 & 33,40 & 20,40 & 29,00 & 42,50 & 28,60 & 32,30 & 13,00 \\
\hline A.G & 52,50 & 43,80 & 16,60 & 64,40 & 73,70 & 14,50 & 25,50 & 19,10 & 25,30 & 20,50 & 25,80 & 25,70 \\
\hline A.C.G & 23,30 & 22,70 & 2,40 & 29,40 & 31,20 & 6,00 & 19,40 & 22,50 & 16,00 & 30,40 & 37,00 & 21,60 \\
\hline M.A.S & 30,10 & 28,30 & 6,20 & 52,60 & 41,90 & 20,30 & 25,90 & 27,00 & 4,30 & 47,10 & 39,60 & 15,90 \\
\hline T.P.R & 64,30 & 53,50 & 16,80 & 104,70 & 80,80 & 22,90 & 61,10 & 46,30 & 24,20 & 89,80 & 71,60 & 20,20 \\
\hline P.R & 37,40 & 31,30 & 16,30 & 48,90 & 46,30 & 5,20 & 32,80 & 35,50 & 8,30 & 43,90 & 46,10 & 5,00 \\
\hline L.A & 50,00 & 41,40 & 17,30 & 52,70 & 70,50 & 33,80 & 40,30 & 35,50 & 11,80 & 41,70 & 47,60 & 14,20 \\
\hline E.E.C & 33,10 & 29,20 & 11,90 & 64,70 & 55,00 & 15,00 & 30,20 & 34,60 & 14,40 & 29,90 & 31,90 & 6,80 \\
\hline D.B.D & 62,10 & 50,70 & 18,30 & 75,40 & 59,50 & 21,10 & 50,20 & 40,10 & 20,00 & 45,50 & 38,10 & 16,30 \\
\hline F.G.L & 32,00 & 29,80 & 7,00 & 49,00 & 55,90 & 14,20 & 28,60 & 33,30 & 16,10 & 44,30 & 46,80 & 5,70 \\
\hline A.L.M & 31,90 & 33,10 & 3,80 & 54,40 & 38,00 & 30,10 & 28,80 & 30,80 & 6,90 & 49,30 & 31,50 & 36,30 \\
\hline S.G & 38,90 & 36,50 & 6,20 & 52,30 & 35,20 & 32,80 & 31,60 & 29,00 & 8,20 & 51,20 & 31,70 & 38,00 \\
\hline M.A.F & 35,00 & 32,00 & 8,57 & 51,50 & 46,00 & 10,68 & 30,00 & 31,00 & 3,30 & 40,10 & 37,40 & 6,73 \\
\hline Médias & 37,32 & 33,81 & 15,28 & 52,91 & 47,85 & 20,87 & 29,82 & 29,46 & 16,12 & 39,15 & 36,93 & 16,07 \\
\hline
\end{tabular}

Legenda: NO: ombro não operado; O: ombro operado

Quando os dados são avaliados dividindo-se os pacientes em dois grupos, para os que operaram o lado dominante a diferença entre os picos de torque, tanto para rotação interna $(180 \%$ s: $p=0,205 ; 60 \%$ s: $p>0,4)$ como para a externa $(180 \%$ s: $\mathrm{p}=0,076 ; 60 \%$ s: $\mathrm{p}>0,4)$ em ambas as velocidades, não foram estatisticamente significativas. No entanto, para os pacientes com o lado operado não dominante, a diminuição no pico de torque do membro afetado foi significativa.

Na comparação entre os déficits de pico de torque para as rotações externa e interna para uma mesma velocidade, a $60 \%$ s a diferença encontrada é estatisticamente significativa para um nível de significância de $5 \%(p=0,046)$, sendo o déficit da rotação interna (20,87\%) superior ao da rotação externa (15,28\%). Para a velocidade de $180 \%$ s, a diferença entre os déficits não é significativa ( $p>0,400)$, pois ambos correspondem a aproximadamente 16\%. Quando comparamos apenas os pacientes com o lado operado não dominante, observamos que o maior déficit é relativo à rotação interna a $180 \%$ s (21,04\%) (Figura 4), enquanto que para o grupo de pacientes que apresentam o lado operado como dominante o maior déficit foi encontrado na rotação interna a $60 \%$ s (23,37\%) (Figura 5).

Os resultados obtidos com as imagens das RNM estão apresentados na Tabela 3.

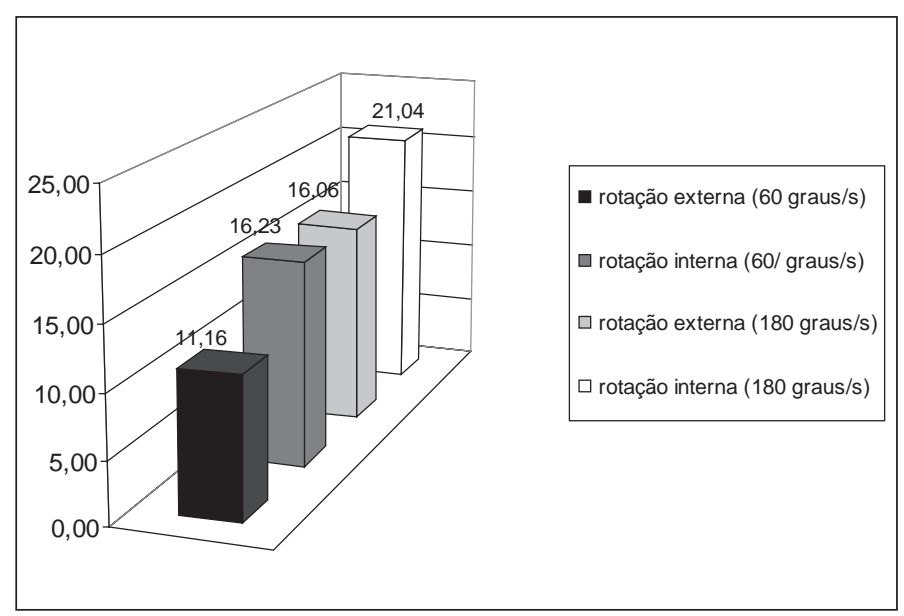

Figura 4 - Percentual de déficit de pico de torque em paciente com lado operado não dominante 


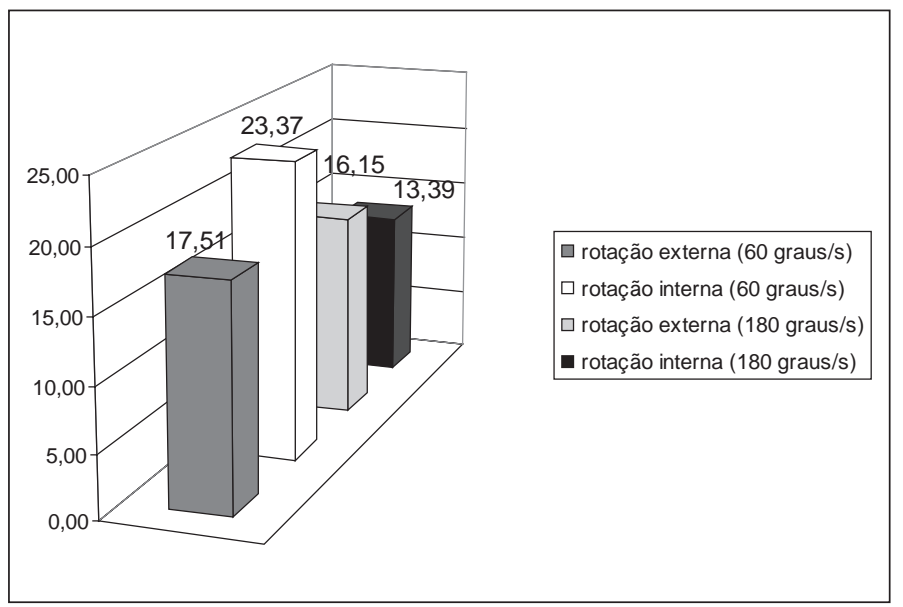

Figura 5 - Percentual de déficit de pico de torque em paciente com lado operado dominante

A espessura do tendão subescapular no membro afetado é significativamente menor que a do lado normal $(\mathrm{p}=$ 0,0005), em média 0,4cm, o que corresponde a um déficit de $19 \%$. Mesmo quando os pacientes são avaliados em grupos distintos, essa diferença também é significativa. Para os pacientes com o lado operado dominante, o déficit apresentado foi de $13,31 \%(p=0,02)$ e de $30 \%$ para os que operaram o lado não dominante $(\mathrm{p}=0,004)$.

A área da seção transversal do subescapular também é significativamente reduzida quando comparada com o lado normal ( $\mathrm{p}=0,0005)$, em média, de $1,7 \mathrm{~cm}^{2}$, o que representa uma diferença de $23 \%$. Para os pacientes com o lado operado dominante o déficit encontrado foi de $14,15 \%$ ( $\mathrm{p}=0,005)$; já para os casos em que o lado operado foi o não dominante, a diferença de área foi de $36,51 \%(p=0,00005)$.

A avaliação da degeneração gordurosa mostrou que 46,7\% apresentavam grau 1, e 33\% não tiveram degeneração gordurosa. Quando os pacientes são avaliados em grupos distintos, nos casos em que o lado dominante corresponde ao membro operado, foram observados apenas grau 0 (33\%) e grau 1 (67\%). Nos pacientes com o lado operado não dominante, 33\% também não apresentaram degeneração. Porém, nesse grupo foram observadas degenerações nos graus 2 (33\%) e 3 (17\%).

Utilizando os sistemas de pontuação de Rowe e da UCLA, observamos excelentes e bons resultados na maioria dos pacientes, com média de 88 e 31,6 pontos, respectivamente (Tabela 1 ).

\section{DISCUSSÃO}

A cirurgia aberta na qual o lábio e o ligamento glenoumeral inferior são reconstituídos é ainda considerado por muitos autores o tratamento de escolha para estabilizar o ombro. Contudo, a cirurgia aberta pode restringir a mobilidade e a força, podendo evoluir para uma osteoartrite secundária ${ }^{(9)}$.

Sachs et al avaliaram a função do músculo subescapular após cirurgia aberta e observaram que $77 \%$ dos pacientes tinham função normal do subescapular e, pelo menos, $80 \%$ da força em comparação com o lado oposto. Todos os pacientes apresentaram um "Belly” Teste negativo ${ }^{(15)}$.

Tabela 3 - Avaliações das imagens de RNM

\begin{tabular}{|c|c|c|c|c|c|c|c|c|c|}
\hline \multirow{2}{*}{ Paciente } & \multirow{2}{*}{ Lado operado } & \multirow{2}{*}{$\begin{array}{l}\text { Lado } \\
\text { dominante }\end{array}$} & \multicolumn{3}{|c|}{ Espessura (cm) } & \multicolumn{3}{|c|}{ Seção transversal $\left(\mathrm{cm}^{2}\right)$} & \multirow{2}{*}{ Goutallie } \\
\hline & & & 0 & NO & Défcit (\%) & 0 & NO & Défcit (\%) & \\
\hline M.A.F & $\mathrm{D}$ & $\mathrm{D}$ & 1,40 & 1,90 & 26,32 & 5,60 & 7,20 & 22,22 & 1 \\
\hline L.L.F & $\mathrm{D}$ & $\mathrm{D}$ & 1,30 & 1,90 & 31,58 & 5,20 & 7,40 & 29,73 & 1 \\
\hline F.G.L & $\mathrm{D}$ & D & 1,30 & 1,10 & $-18,18$ & 5,50 & 5,60 & 1,25 & 1 \\
\hline J.S.D & $\mathrm{D}$ & $\mathrm{D}$ & 1,40 & 1,20 & $-16,67$ & 5,40 & 5,05 & $-6,53$ & 1 \\
\hline M.G & $\mathrm{D}$ & $\mathrm{D}$ & 1,40 & 1,60 & 12,50 & 3,60 & 4,15 & 13,25 & 0 \\
\hline M.A.S & $\mathrm{D}$ & $\mathrm{D}$ & 0,90 & 0,90 & 0,00 & 4,70 & 4,80 & 2,08 & 0 \\
\hline P.R.O & $\mathrm{D}$ & $\mathrm{D}$ & 1,30 & 1,90 & 31,58 & 5,50 & 7,40 & 25,68 & 1 \\
\hline A.G & D & $\mathrm{D}$ & 1,40 & 1,90 & 26,32 & 5,80 & 7,20 & 19,44 & 0 \\
\hline D.B.D & D & $\mathrm{D}$ & 1,40 & 1,90 & 26,32 & 5,90 & 7,25 & 18,62 & 1 \\
\hline S.G & $E$ & $\mathrm{D}$ & 1,80 & 2,20 & 18,18 & 5,85 & 8,95 & 34,64 & 0 \\
\hline C.P & $E$ & D & 1,00 & 2,10 & 52,38 & 4,55 & 7,80 & 41,67 & 1 \\
\hline A.C.G & $E$ & $\mathrm{D}$ & 1,10 & 1,90 & 42,11 & 4,80 & 6,90 & 30,96 & 2 \\
\hline A.F & $E$ & $\mathrm{D}$ & 1,30 & 2,00 & 35,00 & 5,70 & 8,80 & 34,77 & 3 \\
\hline J.A.C & $E$ & $\mathrm{D}$ & 1,40 & 1,60 & 12,50 & 3,35 & 6,10 & 44,99 & 2 \\
\hline P.R & $E$ & $\mathrm{D}$ & 1,70 & 2,10 & 19,05 & 5,90 & 8,60 & 31,39 & 0 \\
\hline Média & & & & & 19,93 & & & 22,94 & \\
\hline
\end{tabular}


Hiemstra et $a^{(16)}$, comparando a força entre pacientes operados por videoartroscopia e cirurgia aberta, não encontraram diferenças estatisticamente significativas na força isocinética para rotação interna e externa em velocidades angulares de 60 e $180 \%$ s.

Em nossa casuística, houve diferença estatisticamente significativa nos picos de torque encontrados para o lado operado e não operado na velocidade de $60 \%$ s, tanto na rotação externa como interna. No entanto, para $180 \%$ s essa situação não é observada. Isso pode ser devido à maior força requerida no teste em menor velocidade. Para os pacientes com lado operado não dominante, as diferenças foram significativas nas duas velocidades angulares, e nos casos em que o lado operado é dominante, as reduções foram insignificantes. Provavelmente, isso ocorre porque o lado dominante é mais utilizado e recupera-se mais apropriadamente.

Em nosso trabalho também observamos que a diferença entre os déficits de pico de torque para as rotações externa e interna a $60 \%$ s foi significativa, sendo o déficit da rotação interna predominante. Para a velocidade de $180 \%$ s, a diferença entre os déficits não foi significativa. Porém, quando comparamos apenas os pacientes com o lado operado não dominante, observamos que o maior déficit é relativo à rotação interna a $180^{\circ}$ s, enquanto que para o grupo de pacientes que apresentam o lado operado como dominante o maior déficit foi encontrado na rotação interna a $60 \%$ s.

Os resultados apresentados em nosso estudo podem eventualmente ser afetados por alguns fatores, pois o teste de força isocinética foi efetuado com o auxílio de um dinamômetro. Portanto, a confiabilidade do teste depende da acurácia do dinamômetro, da reprodutibilidade

\section{REFERÊNCIAS}

1. Neer CS. Cirurgia do ombro. Rio de Janeiro: Revinter; 1995.

2. Lech O. Fundamentos em cirurgia do ombro: ortopedia e reabilitação. Rio de Janeiro: Revinter; 2005.

3. Piluski P. Instabilidade e luxação: conceitos básicos. In: Lech O. Fundamentos em cirurgia do ombro: ortopedia e reabilitação. 2a. ed. Rio de Janeiro: Revinter; 2005. p 395-400.

4. Lech O, Ranzzi A, Bordin F, Faggion M, Zillmer V, Piluski P. Membro superior: abordagem fisioterapêutica das patologias ortopédicas. Rio de Janeiro: Revinter; 2005.

5. Rockwood CA Jr, Matsen FA 3rd, Wirth MA, Lippitt SA. The shoulder. Philadelphia: Saunders; 2004.

6. Snyder SJ. Shoulder arthroscopy. New York: McGraw-Hill; 2002.

7. Lech O. Freitas J R, Piluski P, Severo A. Luxação recidivante do ombro: do papiro de Edwin Smith à capsuloplastia térmica. Rev Bras Ortop. 2005;40(11/12):625-37.

8. Tjoumakaris FP, Abboud J, Hasan SA. Arthroscopic and open Bankart repairs provide similar outcomes.Clin Orthop Relat Res.2006;(446):227-32.

9. Hobby J, Griffin D, Dunbar, M, Boileau, P. Is arthroscopic surgery for stabilization of chronic shoulder instability as effective as open surgery? A systematic review and meta-analysis of 62 studies including 3044 arthroscopic operations. J Bone Joint Surg Br. 2007;89(9):1188-96.

10. Rhee YG, Lim CT, Cho NS. Muscle strength after anterior shoulder stabilization: arthroscopic versus open Bankart repair. Am J Sports Med. 2007;35(11):1859-64. dos parâmetros mensurados, do tipo de protocolo aplicado, dos fatores subjetivos e da motivação do paciente.

As RNM permitiram avaliar que a espessura do subescapular no ombro operado é significativamente inferior à do lado normal, mesmo quando os pacientes são avaliados em grupos distintos (lado operado dominante e não dominante). A diferença encontrada foi de $19 \%$, resultado semelhante ao obtido nos estudos de Tuoheti et al ${ }^{(17)}$ (18,7\%). Na avaliação da área da seção transversal do subescapular também foi observada diminuição significativa no ombro operado, com um déficit de 23\%. Tuoheti et $a l^{(17)}$ encontraram em seu estudo um déficit de $29 \%$.

A avaliação da degeneração gordurosa mostrou que a maioria dos pacientes apresentou bons resultados e somente nos com lado operado não dominante foi observada degeneração nos graus 2 e 3 .

Os resultados apresentados neste trabalho poderiam ser mais representativos se um número maior dos 96 pacientes chamados tivesse comparecido para a avaliação. Apenas 20 pacientes participaram do estudo e os demais não compareceram devido à distância e impossibilidade financeira.

\section{CONCLUSÃO}

A cirurgia aberta na qual o lábio e o ligamento glenoumeral inferior são reconstituídos é ainda considerado por muitos autores o tratamento de escolha para estabilizar o ombro. Contudo, a cirurgia aberta pode restringir a força, fato estatisticamente significativo em nosso estudo. No entanto, encontramos tendências aos melhores resultados nos pacientes que operaram o lado dominante. As RNM também mostraram que há redução significativa na espessura e na área da seção transversal do tendão subescapular, levando a graus variados de hipotrofia.

11. Cahalan TD, Johnson ME, Chao YS. Shoulder strength analysis using the Cybex II isokinetic dynamometer. Clin Orthop Relat Res.1991;(271):249-57.

12. Scheibel M, Nikulka C, Schroeder RJ. Structural integrity and clinical function of the subescapularis musculotendinous unit after arthroscopic and open shoulder stabilization. Am J Sports Med. 2007;35(7):1153-61.

13. Zanetti M, Gerber C, Hodler J. Quantitative assessment of the muscles of the rotator cuff with magnetic resonance imaging. Invest Radiol. 1998;33(3):163-70.

14. Steinbach LS. Magnetic resonance imaging of glenohumeral joint instability. Sem Musculoskelet Radiol. 2005;9(1):44-55.

15. Sachs RA, Williams B, Stone ML, Paxton L, Kuney M. Open Bankart repair: correlation of results with postoperative subscapularis function. Am J Sports Med. 2005;33(10):1458-62.

16. Hiemstra LA, Sasyniuk TM, Mohtadi NG, Hollinshead RM. Open versus arthroscopic shoulder stabilization for recurrent traumatic anterior shoulder instability: a comparison of strength and endurance deficits. [Anais]. In: 10th International Congress of Shoulder and Elbow Surgery (ICSS). Sauípe, Brasil; 2007.

17. Tuoheti $\mathrm{Y}$, Itoi $\mathrm{E}$, Minagawa $\mathrm{H}$, Wakabayashi I, Kobayashi M, Okada K, et al. Quantitative assessment of thinning of the subscapularis tendon in recurrent anterior dislocation of the shoulder by use of magnetic resonance imaging. J Shoulder Elbow Surg. 2005;14(1):11-5. 